\title{
Morphology of early-type galaxies in compact groups. IV. Rose groups
}

\author{
D. Bettoni and G. Fasano \\ Osservatorio Astronomico, Vicolo dell' Osservatorio, 5, I-35122 Padova, Italy \\ Received July 28, 1995; accepted January 23, 1996
}

\begin{abstract}
In the previous papers of this series (Bettoni \& Fasano 1993, 1995 and Fasano \& Bettoni 1994) we presented data relative to early-type galaxies in several Hickson (1982) and Shakhbazyan (1973-1979) Compact Groups. In this paper we present, for the first time, data on early-type galaxies belonging to 9 Rose (1977) compact groups. The selection criteria of these groups make them more compact than the Hickson and Shakhbazyan groups and, for this reason, the Rose groups constitute a good sample for testing the properties of early-type galaxies in environments of extreme (at least apparent) density. Even if the present sample is not a sizeable one (18 objects), we find that morphological and photometric properties of E and S0 galaxies in the Rose groups are fairly consistent with those of galaxies in the Hickson and Shakhbazyan samples. In particular, there is a lack of very flat objects in the ellipticity distribution.
\end{abstract}

Key words: galaxies: compact

\section{Introduction}

In this paper, which is the fourth of a series, we study for the first time the morphological and photometric properties of early-type galaxies in a small sample of compact groups belonging to the Rose (1977) list.

Compact Groups of Galaxies (CGGs) are potentially the densest regions in the universe. However, it remains still open the question if they are accidental superposition of galaxies (Mamon 1986; Hernquist et al. 1995; Ostriker et al. 1995) rather than true physical associations (Hickson \& Rood 1988). Nevertheless, interactions in CGG are observed with a higher frequency than in the field (Hickson 1990). For this reason, their global observed properties (e.g. small velocity dispersions, high projected densities and, then, short crossing times) make them the most suitable objects to be studied in order to improve our knowledge of the properties of galaxies in potentially dense environments and to understand the nature and the true densities of these environments.

Although the Rose CGGs, were defined many years ago (Rose 1977), most of the studies on these groups was devoted to their environmental characteristics (Rose 1979) and little or no work has been done until now to study in detail the individual galaxies belonging to these groups. Almost all studies of galaxies in CGGs are based on the sample given by Hickson (1982). This is mainly due to the

Send offprint requests to: D. Bettoni fact that the Hickson's catalog provides a better defined list of groups over a much larger region of sky $(\sim 60 \%$ of the total) with respect to the other lists.

Actually, the Rose groups differ from the other CGG collections since the adopted selection criteria makes them the most compact systems known, even more compact than the Hickson (HCG) and Shakhbazyan (SHK) groups, although some of the Hickson and Shakhbazyan groups are included in the Rose compilation, (e.g. RCG 13 and 27 coincide with HCG 54 and SHK19 respectively). Therefore, it could be of some interest to study the galaxies belonging to the Rose's groups in order to verify if the increasing density changes systematically the geometrical properties of elliptical galaxies.

In Sect. 2, we give an overview of the observations, referring to the previous papers of the series for more detailed information. Section 3 is devoted to describe briefly each group. In Sect. 4 the main results of this study are summarized.

\section{Observations and data reduction}

The observations were obtained, in the red Cousin's band, by using the $1.82 \mathrm{~m}$ Asiago's telescope equipped with the Tektronix TK512M CCD camera. The log of observations is reported in Table 1 . A detailed description of the system is given in Bettoni \& Fasano (1993, Paper I). The CCD 
Table 1. Log of the observations

\begin{tabular}{c|c|c|c|c|c}
\hline $\begin{array}{c}\text { Rose } \\
\#\end{array}$ & Date & $\begin{array}{c}\text { FWHM }^{\prime \prime} \\
\text { maj. } \times \text { min. }\end{array}$ & $\begin{array}{c}\text { Exp. Time } \\
(\mathrm{sec})\end{array}$ & $\begin{array}{c}\text { Relative error } \\
\text { of sky }(\%)\end{array}$ & $\begin{array}{c}\text { Dwarf } \\
\text { gal. }\end{array}$ \\
\hline 1 & Feb/6/1992 & $1.82 \times 1.75$ & 1200 & 0.8 & 3 \\
5 & Feb/6/1992 & $1.77 \times 1.51$ & 1200 & 0.5 & 2 \\
6 & Feb/6/1992 & $1.64 \times 1.48$ & 1200 & 0.8 & 4 \\
8 & Feb/6/1992 & $1.70 \times 1.50$ & 900 & 0.5 & 1 \\
9 & Feb/7/1992 & $1.72 \times 1.50$ & 1200 & 0.4 & - \\
11 & Feb/7/1992 & $1.78 \times 1.64$ & 1200 & 1.0 & 1 \\
13 & Feb/7/1992 & $1.64 \times 1.47$ & 1200 & 0.7 & 2 \\
14 & Feb/7/1992 & $1.56 \times 1.33$ & 1200 & 0.6 & 1 \\
15 & Feb/7/1992 & $1.60 \times 1.47$ & 750 & 0.9 & 2 \\
\hline
\end{tabular}

has a pixel format of $512 \times 512$ and the $27 \mu$ square pixel size gives a scale of $00^{\prime \prime} 34$ per pixel for a $3^{\prime}$ field of view.

Flat field correction, cosmetics of the chip and sky subtraction have been performed by using the Astronomical Imaging Analysis Package (Fasano 1990; hereafter AIAP). Several sky flats have been averaged and normalized, and finally divided to the images. The sky subtraction has been performed by using a polynomial fit of the regions not occupied by the galaxies and, in general, due to the small diameters of the galaxies in our sample, a third order polynomial has been used. After this, the estimate of the errors has been made following the procedure described in Paper I (see also Fasano \& Bonoli 1990) and we found that the relative errors of the background do not exceed the $1 \%$ (see Col. [4] in Table 1). Unfortunately, during this observing run no photometric standards have been observed and for this reason we give only geometrical information on the galaxies. The magnitudes given for the luminosity profiles are in arbitrary units, being scaled to the sky, to which we decided to assign a fixed value of the surface brightness $\left(\mu_{\mathrm{R}}^{\text {sky }}=21.0\right.$, which is a typical value for the Asiago's sky). The level of the lowest isophote in our profiles varies from 0.8 to 1.0 , in units of the standard deviation of the background. In Fig. 1a the isophotal contours of our frames are shown, all on the same scale and this make more easy to understand the degree of isolation of this kind of compact groups. In Fig. 1b we show a zoomed version of the fields of Rose 6, 11, 13 and 14, which are the faintest of our sample, in order to allow a better resolution of the single galaxies.

The morphological analysis has been performed by means of the AIAP software, allowing to obtain the ellipse fitting of each isophote. This provides us with the luminosity $(\mu)$, ellipticity $(\epsilon)$ and position angle (PA) profiles as a function of the semi-major axis $\left(a^{\prime \prime}\right)$. Moreover, the software provides the $a_{4}$ Fourier coefficient of the residuals, which quantifies the deviations of the isophotal contours from the perfect ellipse, giving information on the disky or boxy shapes of the isophotes. Figure 2 shows the luminosity and geometrical profiles obtained by sampling the surface brightness of the isophotes in steps of 0.25 . In addition all the images have been analyzed by using the Unsharp Masking Technique (see Bettoni \& Fasano 1993; hereafter UMT) to enhance (if present) faint structures.

\section{Description of the groups}

In the following we will give a brief description of each group and of the galaxies we observed. Table 2 reports some basic information on each observed galaxy. In Col. (1) there is the Rose number. In Col. (2) other possible identifications are reported. In Col. (3) we give the morphological type, estimated both from a direct inspection of each image and from the luminosity and geometrical profiles. In Col. (4) we give the effective radius (in arcsecs), derived by fitting the de Vaucouleurs $r^{1 / 4}$ law to the luminosity profiles of early-type galaxies. In Col. (5) we give comments on each galaxy, derived from direct inspection of the frames (before and after UMT). As in the previous papers of this series, we report in Col. (5) of Table 1 the number of non-stellar objects visible on each frame. In Fig. 1 they are marked by an arrow.

\section{Rose 1}

This group is characterized by the pair of galaxies $a$ (a disky elliptical) and $b$ (an early-type spiral with strong bar and bulge components). Although we have no redshift information, the similar size and resolution of the two galaxies suggest that they form a physical pair. On the other hand, a quite evident physical association exists between galaxy $a$ and galaxy $c$, which shows a well-resolved, irregular structure (HII regions?). Finally, galaxy $d$ is likely to be a dwarf E galaxy or a background object.

\section{Rose 5}

This is a very compact group, in which four galaxies are apparently in close interaction with each other. In particular, the interaction between galaxies $b$ (a barred galaxy) and $c$ (probably an S0) is suggested by the asymmetric truncation of the disks, a characteristic commonly 

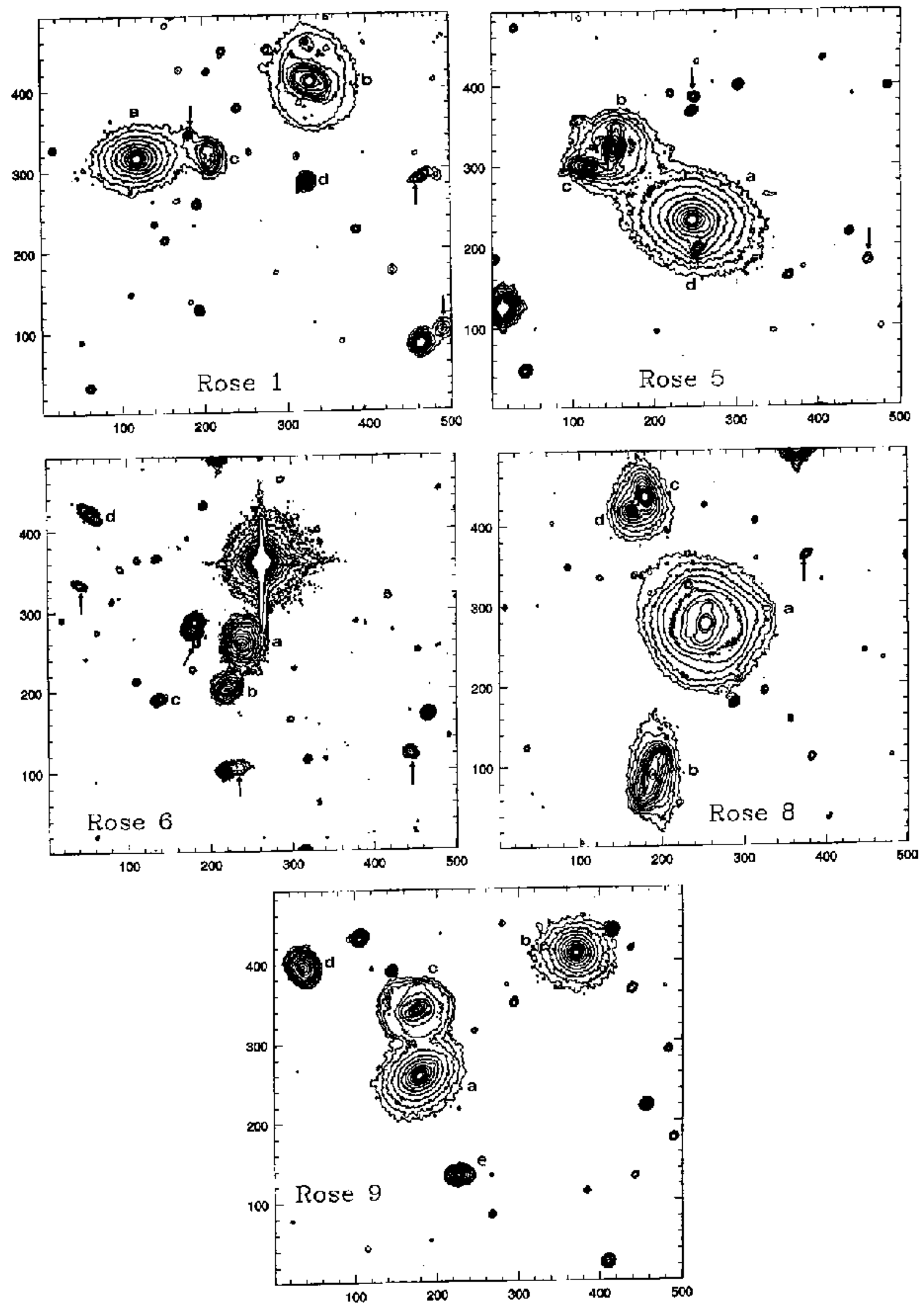

Fig. 1. a) Isophotal contours of our frames. The sampling in surface brightness is in steps of $0.5 \mathrm{mag}^{\mathrm{arcsec}}{ }^{-2}$. All the frames have a size of $170^{\prime \prime} \times 170^{\prime \prime}$. North is at the top, east to the left. The axis coordinates are pixels units. The arrows indicate small diffuse objects listed in Col. (5) of Table 1 

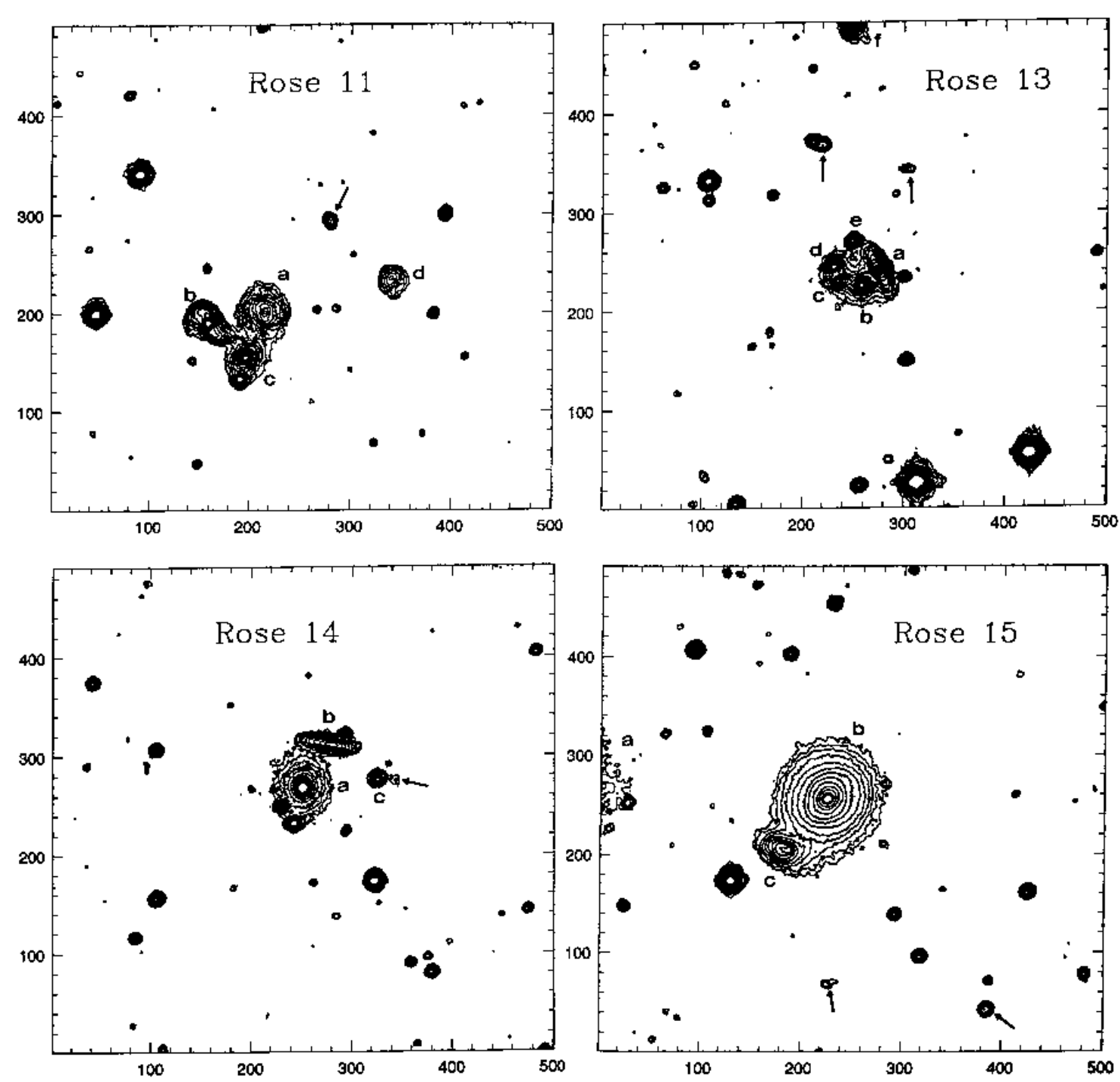

Fig. 1. continued

found in spirals belonging to CGGs (Bettoni \& Fasano 1993). The dominant galaxy $a$ is again a disky elliptical with a faint, low surface brightness companion (galaxy $d$ ) embedded in its halo. The pair of spirals and the dominant elliptical are likely to be connected in the outer regions

\section{Rose 6}

This is not a particularly compact group, composed by the pair $a, b$ and by some very small, additional galaxies (two of them are in the list of Rose) which could actually be background objects. The morphological classification is rather difficult be obtain. Nevertheless, the use of UMT reveals the irregular structure of galaxy $a$ (a barred galaxy?) and a possible signature of interaction with galaxy $b$. Galaxy $d$ shows clearly the presence of a disk and galaxy $c$ is probably the only early-type galaxy of the group. The position of this group coincides with the
IRAS source F11458+2602, but it is impossible to discriminate if the infrared source is the spiral galaxy $a$ or the bright star located near to this galaxy.

\section{Rose 8}

This group shows a chain-like structure (a shape commonly found in CGGs) and it is dominated by the galaxy $a$, which could actually be a foreground object (note, however, the strongly irregular shape of its disk). The two brightest members of the group (galaxies $a$ and $b$ ) are both barred spirals, whereas galaxies $c$ and $d$ are likely to be early-type objects. They seem to be in close interaction with each other, appearing embedded in a common halo. This causes a relevant uncertainty in the geometrical parameters in the region involved by the interaction (note the large error bars in the central part of the geometrical profiles). The UMT technique reveals in galaxy $c$ 

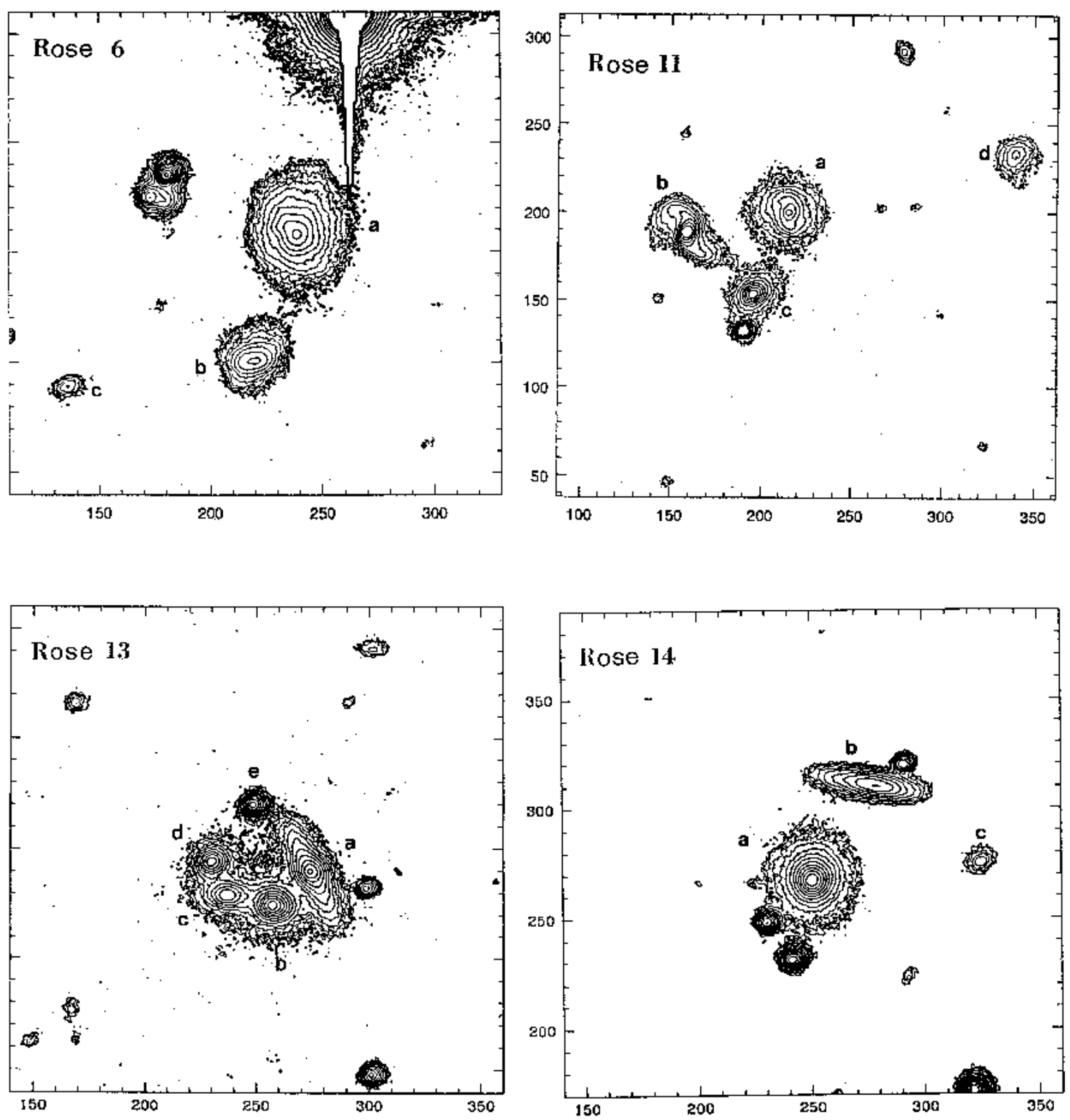

Fig. 1. b) enlargement of the fields of Rose $6,11,13$ and 14 , for all the frames the size is $70^{\prime \prime} \times 70^{\prime \prime}$

(probably an S0) the presence of a stellar disk. On the contrary, the galaxy $d$ looks like a normal elliptical.

\section{Rose 9}

This group also contains a barred galaxy (object c), likely interacting with the disky elliptical $a$ (the UMT reveals in this galaxy the presence of a stellar disk). The small protuberance visible is a star which, as in the previous case, causes an increase of the error bars in the central part of geometrical profiles. This galaxy pair and the galaxy $b$ (a rather normal elliptical) have similar size and resolution. Therefore, although we have no redshift infor- mation, we think these galaxies should be physically associated. On the contrary, we are not convinced that galaxies $d$ and $e$ (probably two poorly resolved spirals) belong to the same association.

\section{Rose 11}

This group is a quite compact one. Besides the triplet $a, b, c$, it includes the more isolated galaxy $d$. The UMT clearly reveals the spiral structure of objects $a$ and $b$ (again barred galaxies). Galaxy $c$ is probably an elliptical, whereas galaxy $d$ (which has very small angular size) 

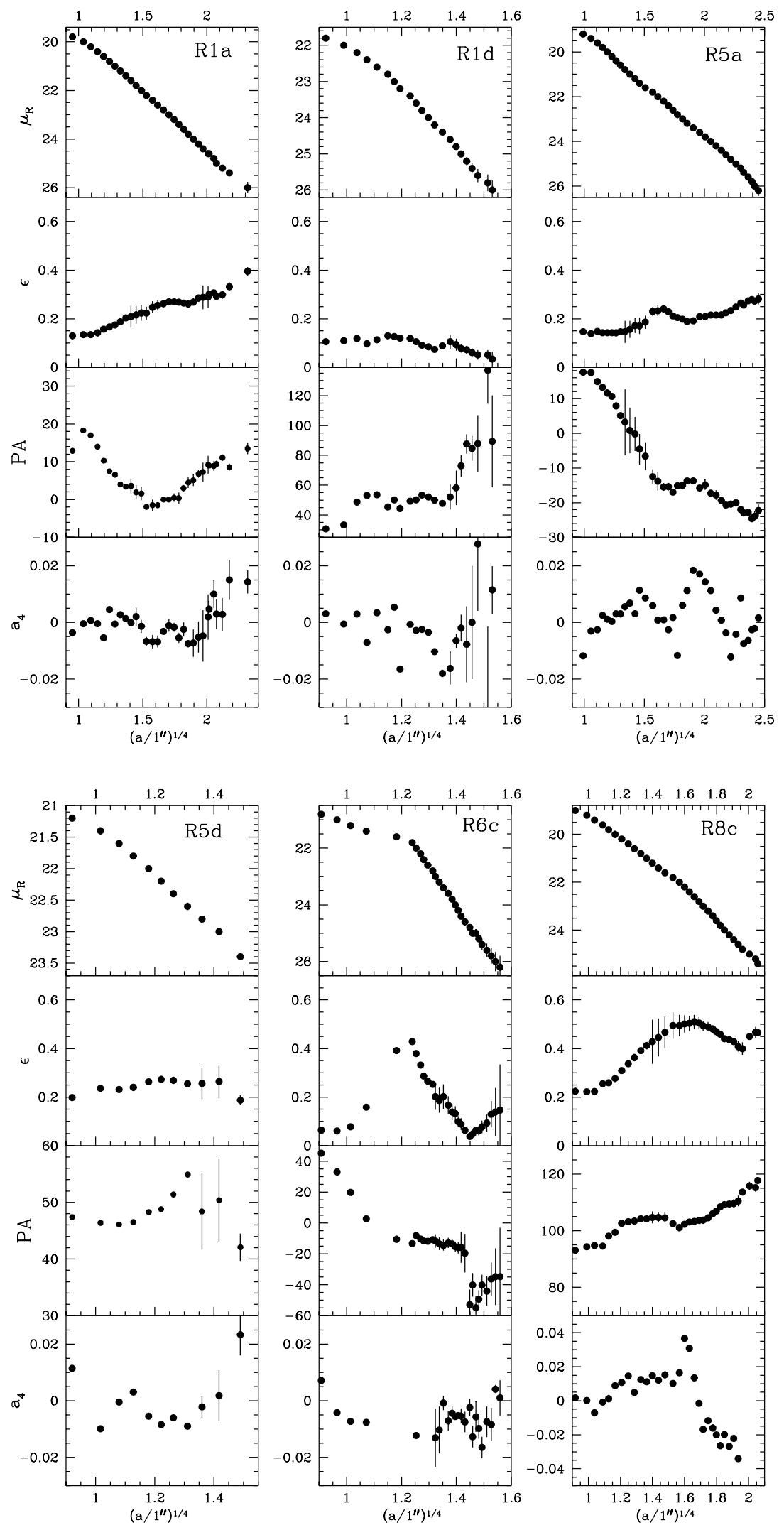

Fig. 2. Surface brightness $\left(\mu_{\mathrm{R}}\right)\left(\mathrm{mag} \operatorname{arcsec}^{-2}\right)$ and geometrical profiles of the $\mathrm{E}$ and S0 galaxies in the sample. The $\mu_{\mathrm{R}}$ profiles derive from the isophotal fit and are relative to the major axis of the fitted isophotes, their normalization assume that $\mu_{\mathrm{R}}^{\text {sky }}=21.0$ for all the frames. The Position Angle is expressed in degrees. The error bars are computed following Fasano \& Bonoli (1990) 

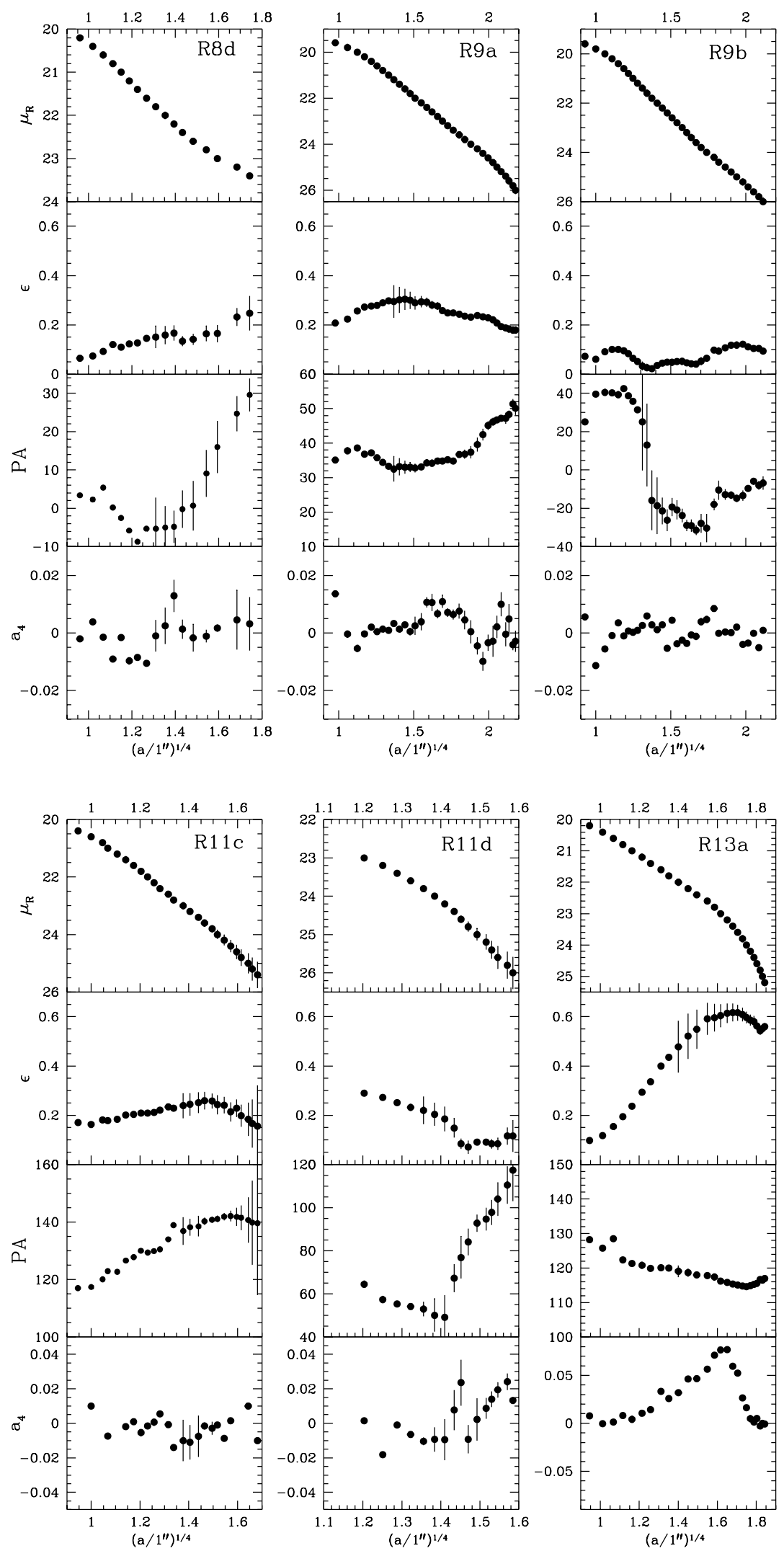

Fig. 2. continued 

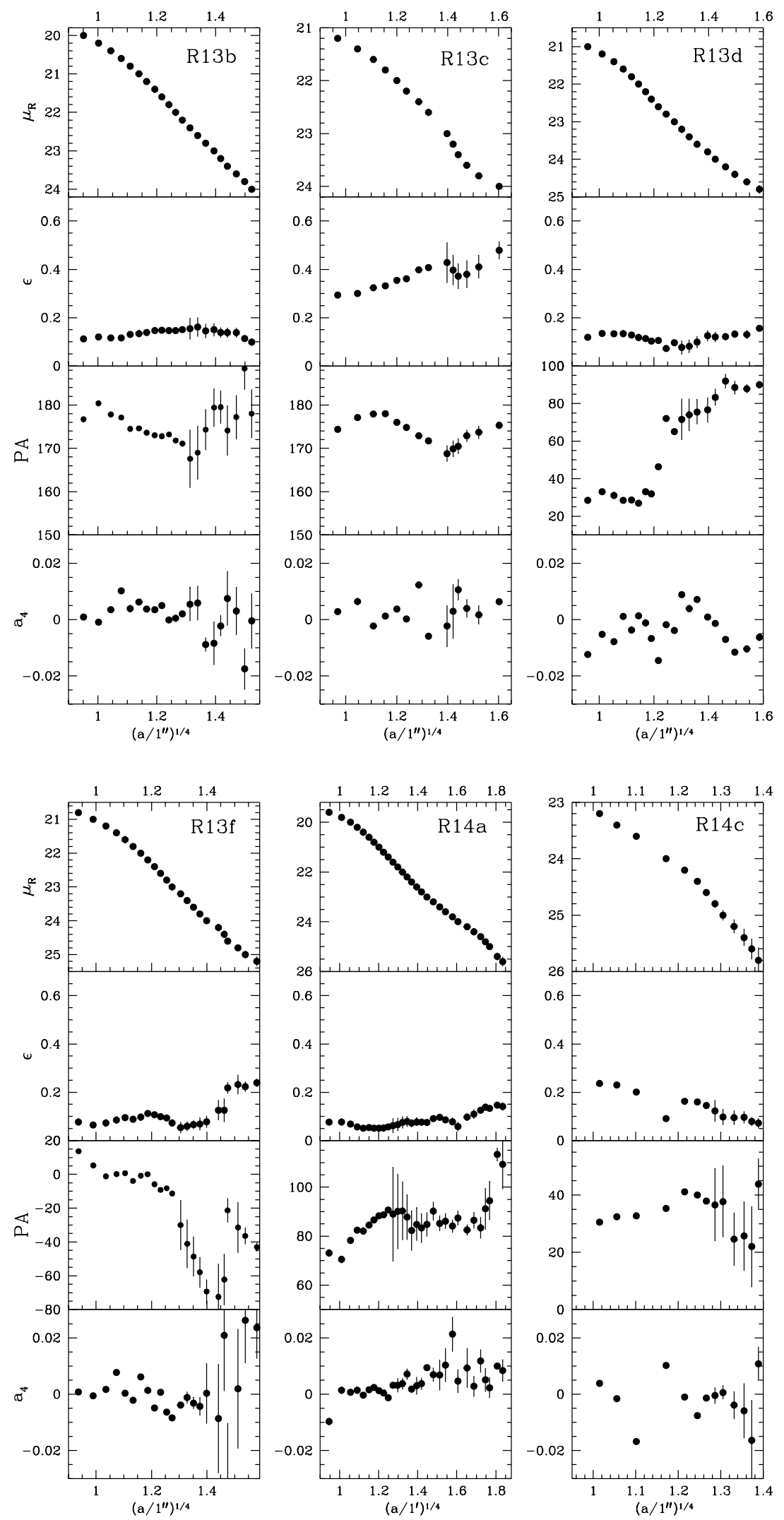

Fig. 2. continued 
Table 2. Properties of individual galaxies

\begin{tabular}{|c|c|c|c|c|}
\hline $\begin{array}{l}\text { Gal. } \\
\#\end{array}$ & $\begin{array}{l}\text { Other } \\
\text { Names }\end{array}$ & Type & $\begin{array}{c}r_{\mathrm{e}} \\
(\operatorname{arcsec})\end{array}$ & comments \\
\hline $\begin{array}{r}1 \mathrm{a} \\
\mathrm{b} \\
\mathrm{c} \\
\mathrm{d}\end{array}$ & & $\begin{array}{l}\mathrm{E} \\
\text { SAB } \\
\text { Irr } \\
\mathrm{E}\end{array}$ & $\begin{array}{l}6.58 \\
- \\
- \\
2.01\end{array}$ & $\begin{array}{l}\text { disky; conn. with } c \\
\text { HII regions? } \\
\text { dwarf or background }\end{array}$ \\
\hline $\begin{array}{c}5 \mathrm{a} \\
\mathrm{b} \\
\mathrm{c} \\
\mathrm{d}\end{array}$ & VV673 & $\begin{array}{c}\mathrm{E} \\
\mathrm{SAB}(\mathrm{r}) \\
\mathrm{S} \\
\mathrm{E} ?\end{array}$ & $\begin{array}{l}8.27 \\
- \\
- \\
-\end{array}$ & $\begin{array}{l}\text { extended halo } \\
\text { truncated lum. prof. } \\
\text { asymmetric disk }\end{array}$ \\
\hline $\begin{array}{r}6 \mathrm{a} \\
\mathrm{b} \\
\mathrm{c} \\
\mathrm{d}\end{array}$ & & $\begin{array}{l}\text { Irr } \\
\text { SB? } \\
\text { E? } \\
\text { S }\end{array}$ & $\begin{array}{c}- \\
- \\
0.70 \\
-\end{array}$ & $\begin{array}{l}\text { IRAS source; bar? } \\
\text { conn. with } a\end{array}$ \\
\hline $\begin{array}{r}8 \mathrm{a} \\
\mathrm{b} \\
\mathrm{c} \\
\mathrm{d}\end{array}$ & U7064 & $\begin{array}{l}\text { SAB }(r) \\
\text { Scd } \\
\text { S0 } \\
\text { E }\end{array}$ & $\begin{array}{c}- \\
- \\
2.63 \\
8.55\end{array}$ & $\begin{array}{l}\text { irregular disk } \\
\text { bar? } \\
\text { conn. with } d\end{array}$ \\
\hline $\begin{array}{c}9 \mathrm{a} \\
\mathrm{b} \\
\mathrm{c} \\
\mathrm{d} \\
\mathrm{e}\end{array}$ & U7114 & $\begin{array}{c}\mathrm{E} \\
\mathrm{E} \\
\mathrm{SAB}(\mathrm{r}) \\
\mathrm{SB}(\mathrm{r}) ? \\
\mathrm{~S}\end{array}$ & $\begin{array}{l}5.64 \\
4.28 \\
- \\
- \\
-\end{array}$ & $\begin{array}{l}\text { disky } \\
\text { conn. with } a \\
\text { background? }\end{array}$ \\
\hline $\begin{array}{r}11 \mathrm{a} \\
\mathrm{b} \\
\mathrm{c} \\
\mathrm{d}\end{array}$ & & $\begin{array}{c}\mathrm{S} \\
\mathrm{S} \\
\mathrm{E} \\
\mathrm{E}\end{array}$ & $\begin{array}{c}- \\
- \\
1.88 \\
3.52\end{array}$ & $\begin{array}{l}\text { bar? } \\
\text { asymmetric disk; bar? } \\
\text { irregular }\end{array}$ \\
\hline $\begin{array}{r}13 \mathrm{a} \\
\mathrm{b} \\
\mathrm{c} \\
\mathrm{d} \\
\mathrm{e}\end{array}$ & VV678 & $\begin{array}{c}\text { S0 } \\
\text { E } \\
\text { S0 } \\
\text { E } \\
\text { E }\end{array}$ & $\begin{array}{l}2.59 \\
1.48 \\
5.13 \\
2.74 \\
2.48\end{array}$ & bump on lum. prof. \\
\hline $\begin{array}{r}14 \mathrm{a} \\
\mathrm{b} \\
\mathrm{c}\end{array}$ & & $\begin{array}{l}\mathrm{E} \\
\mathrm{S} \\
\mathrm{E}\end{array}$ & $\begin{array}{l}1.77 \\
- \\
2.28\end{array}$ & \\
\hline $\begin{array}{r}15 \mathrm{~b} \\
\mathrm{c}\end{array}$ & & $\begin{array}{l}\text { Sa } \\
\text { Irr }\end{array}$ & $\begin{array}{l}- \\
-\end{array}$ & patchy structure \\
\hline
\end{tabular}

is quite difficult to be classified and shows a rather irregular morphology.

\section{Rose 13}

This group (also known as Shakhbazyan 19) is very compact and more or less chain-shaped, the object originally identified as $e$ being indeed a star. The four galaxies seem to be in close interaction and a common envelope is likely to include galaxies $a, b$ and $c$. In galaxies $a$ and $c$ it is well visible a disk structure, whereas no structures are visible in the remaining galaxies, which are too faint to be classified.
Rose 14

This is a very faint group, composed by (at least) three galaxies. In spite of the small angular size, we can confidently classify galaxies $a$ and $b$ as an elliptical and an early type spiral, respectively. Some other non-stellar objects are found close to the group (note in particular the galaxy $c$ ), but it is impossible to establish whether they belong or not to the group.

\section{Rose 15}

The group is composed by four galaxies, but unfortunately our frame includes only two of them. On the East side of the frame the halo of galaxy $a$ is partially visible which is the dominant member of the group and is probably a barred spiral. The two galaxies included in our frame seem to be in close interaction with each other. In particular, the UMT reveals the spiral structure of galaxy $b$ and the irregular shape of its companion galaxy $c$.

\section{Conclusions}

Our galaxy sample (see Table 2 ) is composed by 15 ellipticals (2 are doubtful), $3 \mathrm{~S} 0$ and 16 spiral and irregular galaxies. Similarly to the other compact groups we studied, and also taking into account some uncertainties in the morphological classification, the frequency of early-type galaxies we find in the Rose compact groups $(\sim 54 \%)$ turns out to be in agreement with that observed in the Hickson's ( 55\%, Hickson 1982) and Shakhbazyan's ( 50\%, Bettoni \& Fasano 1995) groups, being quite larger than the corresponding one for field galaxies which is $\sim 35 \%$ (Postman \& Geller 1984).

Table 3 reports the main morphological properties of the early-type galaxies in this sample. In Col. (1) there is the galaxy identification, in Col. (2) the morphological type and in Cols. (3) and (4) there are the isophotal twisting $(\triangle \mathrm{PA})$ and the maximum ellipticity $\left(\epsilon_{\max }\right)$. In Col. (5), the ellipticity profiles are described with symbols $\nearrow, \searrow, \rightarrow$ and $\sim$ which corresponds to profiles increasing, decreasing, flat and irregular (wavy-shaped) respectively. Column (6) describes the $a_{4}$ profiles by means of the symbols D, B, I and F which denote disky, boxy, irregular and flat $a_{4}$ profiles. This classification has been done following the criteria of Bender (1991) which are the widely used ones. Finally, in Col. (7) the possible halo detections are reported.

It is worth noticing that, due to their small angular size, the luminosity and geometrical profiles of galaxies belonging to the groups $6,11,13$ and 14 are very noisy and have low spatial resolution. This also reduces the possibility to detect any type of morphological features (extended halos, dust lanes, multiple nuclei etc..). Actually, among the 15 elliptical galaxies we studied in this paper, none show the presence of dust lanes. This lack of detection is probably due to the fact that in this sample of CGGs 
Table 3. Properties of elliptical and E/S0 galaxies

\begin{tabular}{c|c|c|c|c|c|c}
\hline $\begin{array}{c}\text { Gal. } \\
\#\end{array}$ & Type & $\begin{array}{c}\Delta \mathrm{PA} \\
(\mathrm{deg})\end{array}$ & $\epsilon_{\max }$ & $\begin{array}{c}\epsilon \\
\text { prof. }\end{array}$ & $\begin{array}{c}\text { Isophotal } \\
\text { shape }\end{array}$ & $\begin{array}{c}\text { Halo } \\
\text { detection }\end{array}$ \\
\hline $1 \mathrm{a}$ & $\mathrm{E}$ & 18 & .39 & $\nearrow$ & $\mathrm{D}$ & \\
$1 \mathrm{~d}$ & $\mathrm{E}$ & 85 & .16 & $\underset{\mathrm{B}}{\mathrm{C}}$ & $\mathrm{B}$ & \\
$5 \mathrm{a}$ & $\mathrm{E}$ & 28 & .25 & $\nearrow$ & $\mathrm{D}$ & $\mathrm{H} ?$ \\
$5 \mathrm{~d}$ & $\mathrm{E}$ & 17 & .25 & $\longrightarrow$ & $\mathrm{I} ?$ & $\mathrm{H} ?$ \\
$6 \mathrm{c}$ & $\mathrm{E} ?$ & 43 & .40 & $\sim$ & $\mathrm{F}$ & $\mathrm{H} ?$ \\
$8 \mathrm{c}$ & $\mathrm{S} 0$ & 17 & .50 & $\nearrow$ & $\mathrm{D}$ & $\mathrm{H}$ \\
$8 \mathrm{~d}$ & $\mathrm{E}$ & 108 & .20 & $\longrightarrow$ & $\mathrm{F}$ & $\mathrm{H} ?$ \\
$9 \mathrm{a}$ & $\mathrm{E}$ & 19 & .30 & $\longrightarrow$ & $\mathrm{D}$ & \\
$9 \mathrm{~b}$ & $\mathrm{E}$ & 56 & .10 & $\sim$ & $\mathrm{F}$ & \\
$11 \mathrm{c}$ & $\mathrm{E}$ & 16 & .22 & $\sim$ & $\mathrm{F}$ & \\
$11 \mathrm{~d}$ & $\mathrm{E} ?$ & 68 & .29 & $\sim$ & $\mathrm{D}$ & \\
$13 \mathrm{a}$ & $\mathrm{S} 0$ & 5 & .60 & $\nearrow$ & $\mathrm{D}$ & \\
$13 \mathrm{~b}$ & $\mathrm{E}$ & 21 & .12 & $\stackrel{\mathrm{F}}{\longrightarrow}$ & $\mathrm{F}$ & \\
$13 \mathrm{c}$ & $\mathrm{S} 0$ & 7 & .48 & $\longrightarrow$ & $\mathrm{D} ?$ & $\mathrm{H} ?$ \\
$13 \mathrm{~d}$ & $\mathrm{E}$ & 20 & .12 & $\nearrow$ & $\mathrm{B} ?$ & $\mathrm{H} ?$ \\
$13 \mathrm{f}$ & $\mathrm{E}$ & 51 & .24 & $\sim$ & $\mathrm{F} ?$ & \\
$14 \mathrm{a}$ & $\mathrm{E}$ & 31 & .10 & $\nearrow$ & $\mathrm{D}$ & $\mathrm{H} ?$ \\
$14 \mathrm{c}$ & $\mathrm{E}$ & 33 & .22 & $\searrow$ & $\mathrm{I}$ & \\
\hline
\end{tabular}

Table 4. Statistical properties of elliptical galaxies

\begin{tabular}{l|l|l|l|l|l|l|l}
\hline & Rose & \multicolumn{2}{|c|}{ HCG } & SHK & Field & $\begin{array}{c}\text { Loose } \\
\text { Groups }\end{array}$ & Cluster \\
\hline
\end{tabular}

\begin{tabular}{c|c|c|c|c|c|c|c}
\multicolumn{7}{c}{ Ellipticity Profiles (in \%) } \\
\hline$\epsilon \longrightarrow$ & 27 & 32 & - & 44 & 17 & 12 & 5 \\
$\epsilon \nearrow$ & 33 & 39 & - & 41 & 0 & 55 & 50 \\
$\epsilon \sim$ & 33 & 25 & - & 12 & 0 & 12 & 31 \\
$\epsilon \searrow$ & 2 & 2 & - & 3 & 83 & 20 & 15 \\
Galaxies & 15 & 56 & - & 21 & 12 & 40 & 19 \\
\hline
\end{tabular}

\begin{tabular}{c|c|c|c|c|c|c|c}
\multicolumn{8}{c}{ Isophotal Shape (in \%) } \\
\hline Disky & 33 & 30 & 34 & $25-37$ & 50 & 37 & 20 \\
Boxy & 13 & 9 & 6 & $6-15$ & 19 & 33 & 55 \\
Flat & 40 & 26 & 9 & $12-28$ & 19 & 7 & 15 \\
Irreg. & 13 & 34 & 50 & $22-47$ & 13 & 23 & 10 \\
Galaxies & 15 & 56 & 32 & 21 & 16 & 60 & 20 \\
\hline
\end{tabular}

we are close to the resolution limit which is needed for a possible detection.

As found for ellipticals in other samples of compact groups, this sample also exhibit the classical relation between maximum isophotal twisting and maximum ellipticity (Galletta 1980). Moreover, in spite of its small size this sample has an ellipticity distribution that shows a lack of very flat $(\epsilon>.5)$ objects.

In Table 4 are summarized the percentages, for the different ellipticity profiles and for the isophotal shapes, we found for the elliptical galaxies in the whole sample of CG: Rose (this paper, in Col. 2), Hickson, (Bettoni \& Fasano 1993; Fasano \& Bettoni 1994, labeled as BF in Col. 3a and Zepf \& Whitmore 1993, labeled as ZW in Col. 3b) and finally Shakhbazyan (Bettoni \& Fasano 1995, in Col. 4); for comparison in Cols. 5, 6 and 7 are reported the data for elliptical galaxies in Field, Loose Groups and Cluster respectively. The data for the ellipticity profiles and for the isophotal shape are from di Tullio (1979) and Shioya \& Taniguchi (1993) respectively and are relative to ellipticals only. The last rows gives the total number of galaxies used in the statistics. The data from di Tullio (1979) are useful because in that paper the same classification scheme of the $\epsilon$ profiles used in our papers is adopted. Nevertheless, the comparison with our results must be taken with care for two reasons: (1) the data are obtained from photographic material; (2) the galaxy samples are small (especially for the field galaxies).

The decreasing ellipticity profiles are rare in compact groups ellipticals ( 1 out of 15 in this paper), at variance with the field ellipticals, were they are the most frequent ones. The percentage of increasing and irregular profiles in the present sample is the same $33 \%$ (5 cases) and the flat profiles are $4(27 \%)$. These percentages do not differ significantly from the corresponding ones we found in the previous papers of this series. From Table 4 we note that ellipticals in clusters show increasing or irregular $\epsilon$ profiles, whereas decreasing or flat profiles are not frequent.

Concerning the boxiness, we found only two cases (13\%) of boxy isophotes in the Rose compact groups. This could be consistent with the field environment but, in spite of the small sample size ( 15 objects), it is different from the analogous fraction in clusters. The most common shapes are the regular (6 objects, $40 \%)$ and the disky (5 objects, $33 \%$ ) ones, whereas irregular $a_{4}$ profiles are found in two cases $(13 \%)$.

These data add new pieces to the mosaic of the properties of early-type galaxies in CGGs. Most of the results found in our previous works are confirmed. In particular, $\mathrm{E}$ and $\mathrm{S} 0$ galaxies in the present sample show fewer signatures of boxiness with respect to galaxies of the same type in randomly selected samples. Also the ellipticity distribution is different if compared with that obtained for randomly selected samples of ellipticals (Fasano \& Vio 1991), and finally a scarcity of decreasing ellipticity profiles is found. In spite of these statistical peculiarities, the luminosity profiles of elliptical galaxies in this sample are in general well represented by $r^{1 / 4}$ laws. Again, this is in agreement with previously studied samples of CGGs and lead us to the conclusion that the Rose compact groups are very similar to the Hickson's and Shakhbazyan's groups. The only thing we wish to note in the present sample is the abnormally large percentage of barred objects among the spiral galaxies. However, we stress again that the poor statistics prevents us to draw any firm conclusion. 
The fact that all available photometric studies on early-type galaxies in CGGs (Papers I-III and Zepf \& Whitmore 1993) show a similarity in their morphological properties (independently on the adopted selection criteria) could be an indication in favor of the hypothesis that compact groups are true physical entities, but (at this stage) we cannot exclude that often they might be produced by chance alignments along the line of sight, between single, binary and/or triple galaxies within loose groups or the nearby field.

Acknowledgements. We acknowledge the referee G. Mamon whose comments contributed to improve the paper

\section{References}

Bender R., 1991, in "Morphological and Physical Classifications of Galaxies". In: Longo G., Capaccioli M. \& Busarello G. (eds.). Dordrecht: Kluwer, p. 357

Bettoni D., Fasano G., 1993, AJ 105, 1291 (Paper I)

Bettoni D., Fasano G., 1995, AJ 109, 32 (Paper III) di Tullio G., 1979, A\&AS 37, 591

Fasano G., 1990, Internal Report of the Padova Astronomical Observatory (AIAP)

Fasano G., Bettoni D., 1994, AJ 107, 1649 (Paper II)

Fasano G., Bonoli C., 1990, A\&A 234, 89

Fasano G., Vio R., 1991, MNRAS 249, 629

Galletta G., 1980, A\&A 181, 179

Hernquist L., Katz N., Weinberg D.H., 1995, ApJ 442, 57

Hickson P., 1982 ApJ 255, 382

Hickson P., 1990, in Paired and Interacting Galaxies IAU Coll. 124. In: Sulentic J.W., Keel W.C. and Telesco C.M. (eds.) NASA Conf. Publ. 3098, p. 77

Hickson P., Rood H.J., 1988, ApJ 331, L89

Mamon G.A., 1986, ApJ 307, 426

Ostriker J.P., Lubin L.M., Hernquist L., 1995, ApJ 444, L61

Postman M., Geller M.J., 1984, ApJ 281, 95

Rose J.A., 1977, ApJ 211, 311

Rose J.A., 1979, ApJ 231, 10

Shakhbazyan R.K., 1973, Afz 9, 495

Shioya Y., Taniguchi Y., 1993, PASJ 45, L39

Zepf S.E., Whitmore B.C., 1993, ApJ 418, 72 